\title{
Anterior cruciate ligament autograft maturation on sequential postoperative MRI is not correlated with clinical outcome and anterior knee stability
}

\author{
Patricia M. Lutz ${ }^{1} \cdot$ Andrea Achtnich $^{1} \cdot$ Vincent Schütte $^{2} \cdot$ Klaus Woertler $^{3} \cdot$ Andreas B. Imhoff $^{1}\left(\right.$ Lukas Willinger $^{1}$
}

Received: 14 May 2021 / Accepted: 18 October 2021 / Published online: 5 November 2021

(C) The Author(s) 2021

\begin{abstract}
Purpose Magnetic resonance imaging (MRI) signal intensity is correlated to structural postoperative changes of the anterior cruciate ligament (ACL) autograft. The purpose of this study was to investigate the ACL autograft maturation process via MRI over 2 years postoperatively, compare it to a native ACL signal and correlate the results with clinical outcome, return to preinjury sports levels, and knee laxity measurements.

Methods ACL autograft signal intensity was measured in 17 male patients (age, $28.3 \pm 7.0$ years) who underwent ACL reconstruction with hamstring autograft at 6 weeks, 3-, 6-, 12-, and 24 months postoperatively by 3 Tesla MRI. Controls with an intact ACL served as control group (22 males, 8 females; age, 26.7 \pm 6.8 years). An ACL/PCL ratio (APR) and ACL/muscle ratio (AMR) was calculated to normalize signals to soft tissue signal. APR and AMR were compared across time and to native ACL signal. Clinical outcome scores (IKDC, Lysholm), return to preinjury sports levels (Tegner activity scale), and knee laxity measurement (KT-1000) were obtained and correlated to APR and AMR at the respective time points. Results The APR and AMR of the ACL graft changed significantly from the lowest values at 6 weeks to reach the highest intensity after 6 months $(p<0.001)$. Then, the APR and AMR were significantly different from a native ACL 6 months after surgery $(p<0.01)$ but approached the APR and AMR of the native ACL at 1 - and 2 years after surgery $(p<0.05)$. The APR changed significantly during the first 2 years postoperatively in the proximal $(p<0.001)$, mid-substance $(p<0.001)$, and distal $(p<0.01)$ intraarticular portion of the ACL autograft. A hypo-intense ACL MRI signal was associated with return to the preinjury sports level $(p<0.05)$. No correlation was found between ACL MRI graft signal and clinical outcome scores or KT-1000 measurements.

Conclusion ACL grafts undergo a continuous maturation process in the first 2 years after surgery. The ACL graft signals became hyper-intense 6 months postoperatively and approximated the signal of a native intact ACL at 12- and 24 months. Patients with a hypo-intense ACL graft signal at 2 years follow-up were more likely to return to preinjury sports levels. The results of the present study provide a template for monitoring the normal ACL maturation process via MRI in case of prolonged clinical symptoms. However, subjective outcome and clinical examination of knee laxity remain important to assess the treatment success and to allow to return to sports.
\end{abstract}

Level of evidence III.

Keywords Anterior cruciate ligament $\cdot$ Graft healing $\cdot$ Ligamentization $\cdot$ MRI $\cdot$ Hamstring autograft $\cdot$ Graft maturation

Patricia M. Lutz and Andrea Achtnich have contributed equally to this work.

The research was performed at the Department for Orthopedic Sports Medicine, Technical University Munich, Germany.

Andreas B. Imhoff

imhoff@tum.de

Extended author information available on the last page of the article

\section{Introduction}

Anterior cruciate ligament (ACL) reconstruction aims to restore knee stability and regain normal knee function. This allows safe return to activity and reduce the risk of re-injuries. Despite advances in surgical techniques, early ACL re-tears are still a major concern [30, 32, 37]. One reason for early re-ruptures is biological failure due to the remodelling process [29]. The graft maturation process (also 
known as ligamentization) during the early postoperative phase negatively affects the biomechanical properties of the graft $[36,41,46]$. The decrease of mechanical strength during the first period is of clinical importance as it puts the graft at risk of not meeting its demands $[2,18,21,23,40,46$, 47]. Hence, assessing ACL graft maturity before returning to sports is of high clinical interest and magnetic resonance imaging (MRI) has been shown to be a feasible option [11, 12]. Animal models showed that MRI signal intensity is significantly correlated to structural properties of the ACL graft [5, 46]. A high signal intensity on MRI has been linked to decreased mechanical properties such as low load to failure [46]. In recent years, several MRI studies showed that signal intensity changes of the ACL graft are correlated with the progress of graft maturation in human patients $[9,11,27$, 43]. ACL graft MRI signal intensity increases during the first 6 months followed by a subsequent decline [16, 42, $45,48]$. These findings are in line with histological findings which suggest a high vascularity and cell proliferation process during the early remodelling phase starting after three to nine months postoperatively [1, 29, 33, 34, 44].

However, current studies evaluating the MRI signal changes of ACL autografts and their correlation to clinical outcome and knee laxity remain inconclusive $[6,12,16,17$, 35]. It is further unknown how ACL autograft MRI signal changes compare to a native ACL MRI signal $[11,12,16$, 17, 24]. Concerning sports activities, reported rates of return to sports after ACL reconstruction (ACL-R) vary in the literature $[4,19,22,31]$. Previous research stated a low return to preinjury sports levels 1 year after ACL-R [15], whereas a clear increase 2 years postoperatively was described [19, 22]. An additional aspect remains the significantly increased incidence rate of second injuries to the ACL autograft in the first year postoperatively [23].

Therefore, the purpose of this study was to investigate (1) the MRI signal intensity changes of hamstring ACL autografts over the first 2 years postoperatively and compare them to a native ACL and (2) to correlate the MRI signal with clinical outcome scores, return to preinjury sports levels and knee laxity measurements. It was hypothesized that (1) the ACL would mature over time at 1 and 2 years postoperatively and would approximate the signal of a native ACL at final follow-up and (2) a higher grade of graft maturation (hypo-intense MRI signal) would positively correlate with better clinical outcome, return to preinjury sports levels, and knee stability.

\section{Materials and methods}

Ethical approval was obtained from the Ethics Committee of the technical University Munich (329/19S). All procedures performed were in accordance with the ethical standards of the institutional and/or national research committee and with the 1964 Declaration of Helsinki and its later amendments or comparable ethical standards. All patients gave their written informed consent to participate in this investigation.

\section{Patients}

Patients were eligible for inclusion in this study after undergoing unilateral four-strand autograft ACL-R (semitendinosus \pm gracilis tendon) following ACL rupture diagnosed by clinical examination and MRI scans and confirmed arthroscopically at time of surgery. Patients were excluded from the study if they had a history of previous surgery at the index knee, a cartilage injury or ligamentous injuries other than ACL rupture including collateral ligaments and posterior cruciate ligament (PCL). Patients with contralateral knee injuries were excluded. Concomitant meniscal lesions were not an exclusion criterion. Baseline demographic variables including age and gender were manually collected using clinical documentation.

30 patients without previous or acute ligamentous knee injuries were included and served as age-matched control group for defining an intact native ACL MRI signal baseline at one time point. Data were collected prospectively in the patient's cohort but retrospectively compared with the control group.

\section{Knee laxity measurements}

All patients underwent clinical examination of the knee including previously validated KT-1000 arthrometer measurements (MEDmetric, San Diego, CA, USA) by a knee trained orthopaedic surgeon (blinded) at 12- and 24 months after surgery. The KT-1000 arthrometer measurements were performed using a standardized $134 \mathrm{~N}$ anterior drawer force at $30^{\circ}$ knee flexion and side-to-side differences were recorded.

\section{Clinical outcomes}

Patient-reported clinical outcome scores were measured using the subjective Form of the International Documentation Society Score (IKDC) and the Lysholm score preoperatively and at 6-, 12-, and 24-month postoperatively $[13,39]$. Tegner activity scale (TAS) was used to measure postoperative sports participation at 1- and 2- years follow up to determine if a patient achieved the same sports level compared to the pre-injury state. The patient was considered 
to have returned to the same sports level when preinjury and postoperative TAS revealed the same value.

\section{Imaging procedures}

Postoperative MRI examination were performed at 6-weeks, 3-, 6-, 12- and 24- months after ACL reconstruction using a 3 Tesla whole-body scanner (Ingenia, Philips Healthcare, The Netherlands) and a dedicated 8-channel knee coil to continuously monitor the ACL graft maturation process. On sagittal high-resolution proton-density (PD) intermediateweighted MR images parallel to ACL (repetition time $5000 \mathrm{~ms}$, echo time $45 \mathrm{~ms}$, field of view $160 \mathrm{~mm}$, in-plane resolution $0.4 \times 0.4 \mathrm{~mm}$, slice thickness $2.5 \mathrm{~mm}$, acquisition time 4:15 $\mathrm{min}$ ) the MRI signal intensity was measured manually in five regions of interest (ROI): intraarticular proximal ACL, mid-substance ACL, distal ACL, mid-substance PCL, medial head of the gastrocnemius muscle (Fig. 1). All measurements were performed on a conventional PACS system (Sectra Medical Systems, Sweden). A standardized $4 \mathrm{~mm}$ diameter circle was drawn at each ROI and the average MRI signal intensity was automatically calculated by the imaging software [28, 38]. The MRI signal outcome was not affected by contrast and brightness settings. To normalize the signal intensity, an ACL/PCL signal ratio (APR) and an ACL/muscle ratio (AMR) were calculated (each for the proximal, mid-substance, and distal ACL portion) by dividing the ACL signal intensity by the signal of the intact PCL or the medial head of the gastrocnemius muscle. Muscle tissue was chosen as a second internal reference to avoid systematic measurement error caused by variation due to possible degenerative changes of the PCL. We believe that not including the measurement of background noise (proton density of extracorporeal hydrogen atoms) the reliability and accuracy is increased. The APR and AMR measurements were performed independently by two knee trained orthopaedic surgeons (blinded) and the average of both measurements was used for analysis.

\section{Surgical technique}

Arthroscopic ACL-R was performed in single-bundle technique with an autologous hamstring graft (semitendinosus \pm gracilis tendon) and anatomic femoral and tibial tunnel placement. The femoral tunnel was drilled via an anteromedial portal according to the diameter of the ACL autograft. A cortical suspension device (ACL TightRope, Arthrex, Naples, USA) was used for femoral graft fixation and a bioabsorbable interference screw (Arthrex, Naples, USA) was used for tibial fixation. Concomitant meniscus lesions were treated by either all-inside meniscus suture devices (FastFix, Smith\&Nephew, London, UK) or inside-out technique.

\section{Postoperative rehabilitation}

The postoperative protocol after ACL-R consisted of 6 weeks of partial weight-bearing on crutches with limitation in range of motion (ROM): in the first 6 weeks postoperatively, flexion was only allowed to $90^{\circ}$. After 6 weeks, ROM was no longer limited. A brace (Medi M4, Medi Bayreuth, Germany) was provided for at least 12 weeks. After

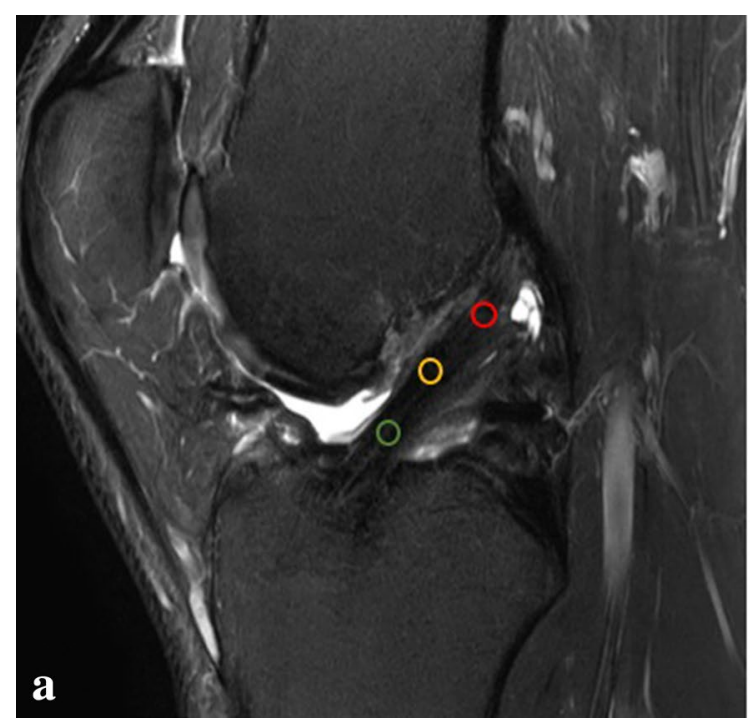

Fig. 1 Magnetic resonance imaging signal intensity was measured within a standardized $4 \mathrm{~mm}$ diameter circle at the respective region of interest at (A) the proximal (red), mid-substance (yellow) and dis-

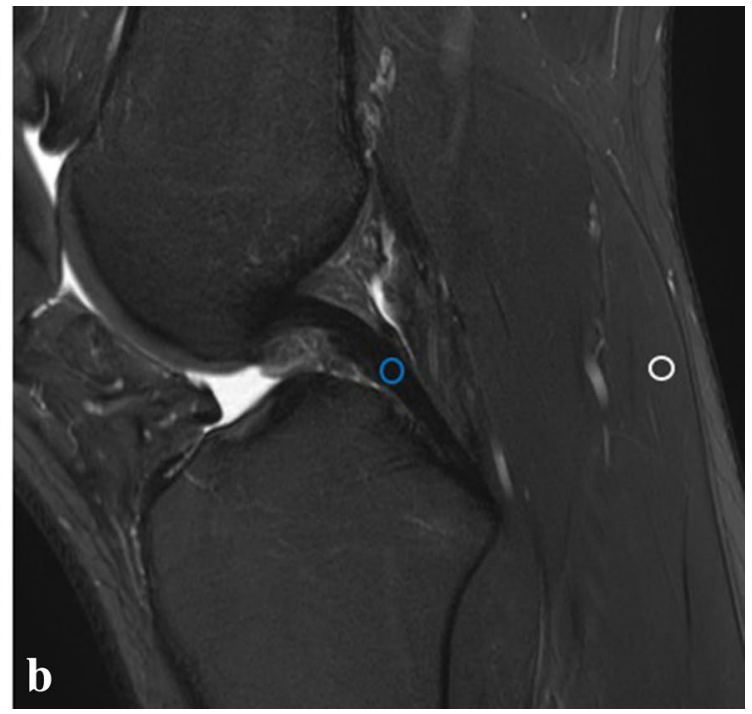

tal (green) portion of the anterior cruciate ligament autograft and (B) at the mid-substance of the posterior cruciate ligament (blue) and the medial head of the gastrocnemius (white) 
8 weeks, sports activities, such as swimming, walking, and cycling and after 12 weeks running were allowed. Return to sport-specific training was allowed after 6 months and full return to contact and/or pivoting sports activities after at least 9 months postoperatively.

\section{Statistical analysis}

Statistical analysis was performed using SPSS software Version 23 (IBM, Armonk, New York, USA) and Microsoft Excel Version 2019 (Microsoft, Redmond, Washington, USA). Descriptive statistics are presented as mean \pm standard deviation (SD) for all continuous variables allowing one decimal. Frequencies $(n, \%)$ were used to obtain descriptive statistics for all categorical variables allowing no decimal. Pearson's correlation coefficient $(r)$ was used to calculated correlations between MRI autograft signal ratios (APR or AMR) and clinical outcomes scores and KT-1000 measurements at the respective time points. The minimal clinically important difference (MCID) for IKDC was set at 9 points, for Lysholm score at 10 points, whereas the minimal detectable change (MDC) for TAS was set at 1 point [7]. Shapiro-Wilk test was used for continuous variables to confirm data normality. Depending on data normality, students $t$-test or Mann-Whitney $U$ test were used to find differences in APR and AMR between ACL grafts and native ACLs. Oneway analysis of variance (ANOVA) with Bonferroni correction was used to describe differences in APR and AMR over time. Interclass correlation coefficient (ICC) was calculated for inter-rater reliability between the two readers for APR and AMR measurements. Based on previous research [17], a sample size calculation resulted in a total number of 44 patients with a 2:1 group allocation ratio (29:15 subjects) to detect a difference in APR MRI signal intensity of 30\% between ACL autograft and the native ACL at a critical p-value of 0.05 with an actual power of $86.6 \%$.

\section{Results}

Seventeen male patients with a mean age of $28.3 \pm 7.0$ years who underwent ACL-R were included in the study. All patients were recreational athletes and none of the patients were lost to follow up for the entire study period of 2 years. One patient suffered a contralateral ACL injury 1.5 years after initial ACL-R.

The control group consisted of 30 patients (22 male and 8 female) with an intact ACL. These patients were treated for either meniscal tears, chondral lesions, or patellofemoral instability and had a ligamentous stable knee. The mean age of the control group was $26.7 \pm 6.8$ years (Table 1 ).
Table 1 Demographic data comparing the patient group with the control group

\begin{tabular}{llll}
\hline & Patient group & Control group & $p$ value \\
\hline Age & $28.3 \pm 7.0$ & $26.7 \pm 6.8$ & n.s \\
Gender (male/female) & $17 / 0$ & $22 / 8$ & $p=0.038$ \\
BMI $\left(\mathrm{kg} / \mathrm{m}^{2}\right)$ & $25.3 \pm 3.0$ & $23.8 \pm 2.7$ & n.s \\
Affected side (right/left) & $5 / 12$ & $14 / 16$ & n.s \\
Pre-injury Tegner score & 6 (range 4-9) & - & n.a \\
\hline
\end{tabular}

$B M I$ body mass index, n.s. not significant, n.a. not applicable

The ICC values for reliability of MRI measurements were 0.834 (95\% CI 0.776-0878) for APR and 0.871 (95\% CI $0.825-0.906)$ for AMR indicating excellent reliability.

\section{Native ACL}

The ACL/PCL MRI signal ratio (APR) of the native intact ACL was $2.6 \pm 1.0$ at the proximal portion, $2.6 \pm 0.9$ at the mid-substance and $3.4 \pm 1.4$ at the distal portion, meaning that the signal of the native ACL was 2.6-3.4 times more intense than the PCL. The ACL/muscle ratio (AMR) was $0.4 \pm 0.2$ proximally, $0.4 \pm 0.2$ at mid-substance and $0.6 \pm 0.2$ distally, meaning that the native ACL had only $40-57 \%$ MRI signal intensity compared with the medial head of the gastrocnemius muscle. The APR and AMR of the distal ACL were significantly higher than the APR and AMR of the proximal and mid-substance ACL (APR $p<0.05$, AMR $p<0.01$, see also Fig. 3F).

\section{APR measurements}

The APR of the ACL autograft over the postoperative period is shown in Fig. 2A. The APR changed significantly during the first two years postoperatively in the proximal $(p<0.001)$, mid-substance $(p<0.001)$, and distal $(p<0.01)$ intraarticular portion of the ACL autograft. The distal ACL portion showed the lowest APR and was significantly different to the mid-substance $(p<0.05)$ ACL graft Sects. 3 months postoperatively. There was no difference in APR between the different measurement locations at 1- and 2 years follow up.

The proximal ACL graft underwent a significant MRI signal change from 6 weeks to 6 months after surgery and showed the highest signal intensity at this time point $(p<0.001)$. The APR subsequently declined to approximate the APR of a native proximal ACL at 1 and 2 years. The proximal APR was significantly lower than a native ACL at 6 weeks $(p<0.001)$ and higher at 6 months $(p<0.05)$ after surgery.

Similarly, the APR of the mid-substance ACL graft ran through a significant transformation from 6 weeks to 2 years 


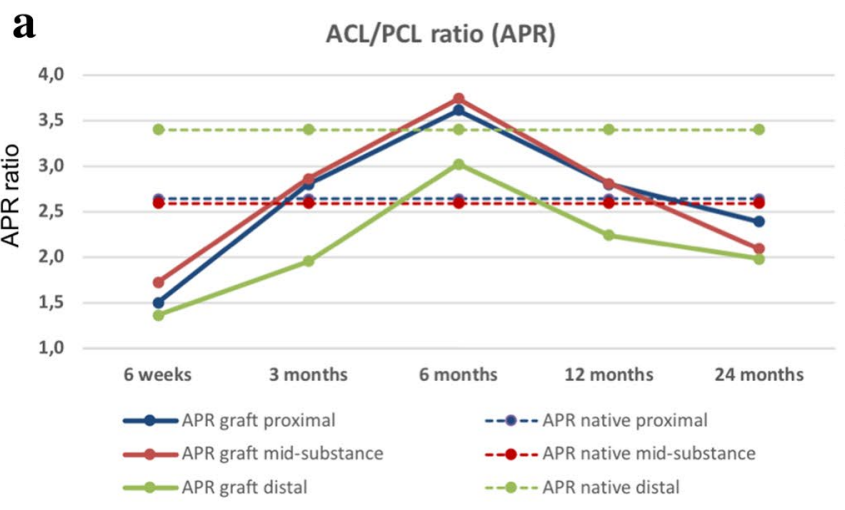

Fig. 2 The ACL autograft underwent significant transformation during the first 2 years after ACL reconstruction. This is shown by a significant increase in APR (A) and AMR (B) from 6 weeks to 6 months after surgery before dropping to reach values of native intact ACL at 1 and 2 years postoperatively. The distal portion of the native ACL

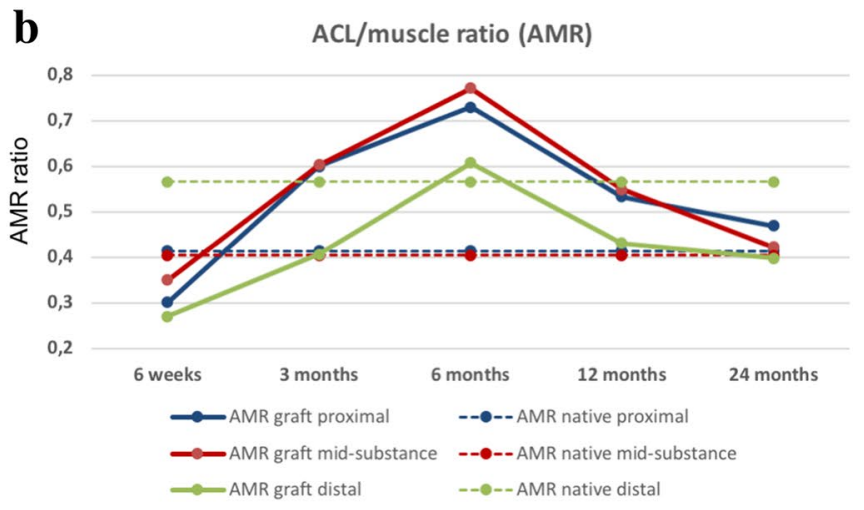

exhibits a significantly more hyperintense signal compared to the proximal and mid-substance portion. The distal part of the ACL autograft shows a significantly lower APR and AMR at 3 months compared to the mid-substance
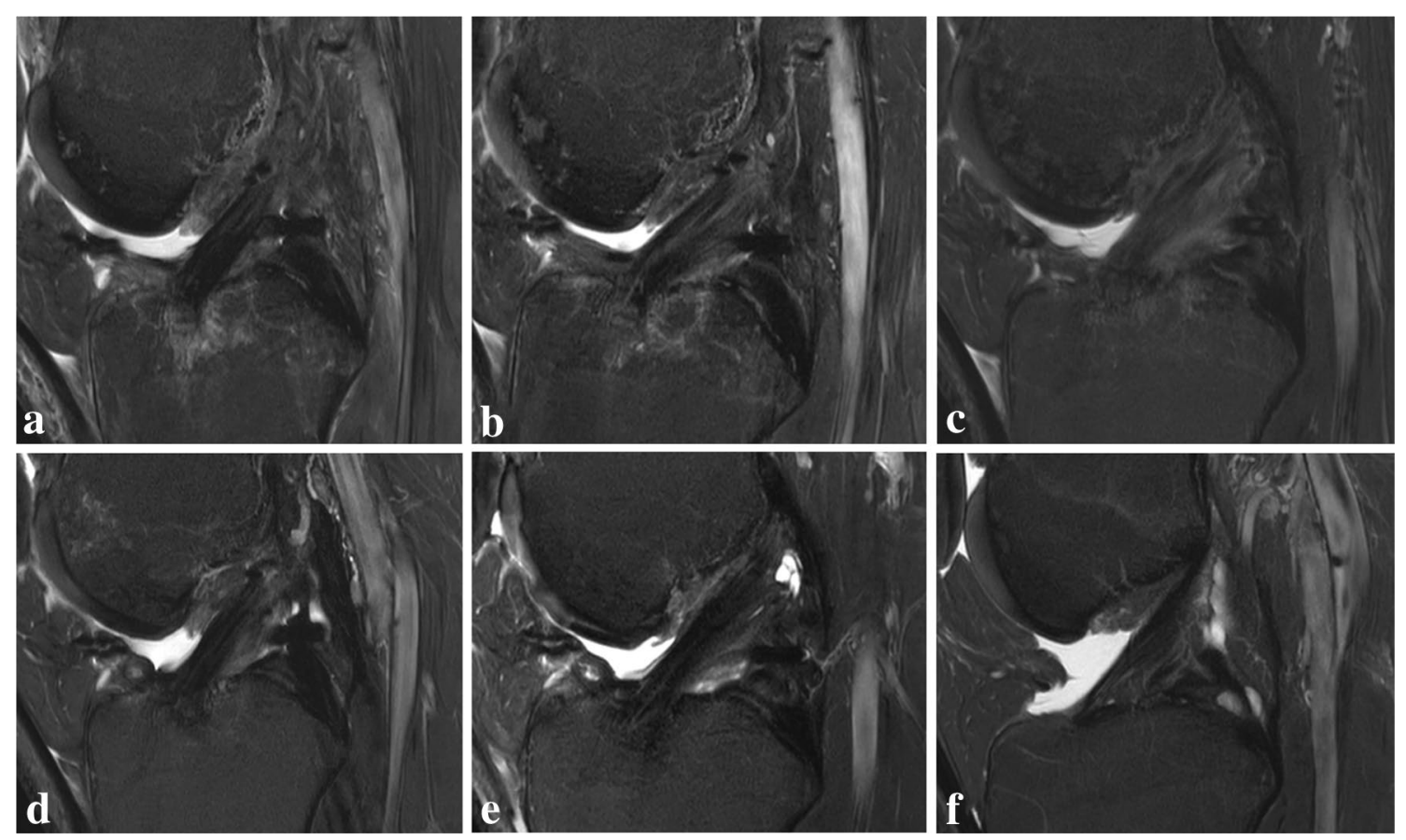

Fig. 3 The maturation process of the anterior cruciate ligament autograft exhibits a significant MRI signal change from hypo-intense at (A) 6 weeks postoperatively to more hyper-intense at (B) 3- and (C) 6-months after ACL-R indicating greater disorganization of collagen tissue and higher water content. The MRI signal intensity decreases

postoperatively. While the APR 6 months after ACL-R is significantly different from a native mid-substance ACL $(p<0.01)$, the APR after 1 and 2 years showed a similar MRI signal compared with a native ACL.

APR of the distal section of the ACL graft showed a significant rise within the first 6 months postoperatively thereafter at (D) 1- and (E) 2-years post ACL reconstruction to approximate the signal of a native ACL $(\mathbf{F})$. The distal native ACL revealed a more hyper-intense signal compared to the proximal and mid-substance ACL

$(p<0.05)$ before dropping gradually at 1 and 2 years. The APR of distal section of the ACL autograft was significantly lower than the native ACL at all time points except from 6 months postoperatively (all $p<0.01$ ). 


\section{AMR measurements}

The AMR of the ACL autograft over the postoperative period is shown in Fig. 2B. The AMR changed significantly at the proximal $(p<0.001)$, mid-substance $(p<0.001)$, and distal $(p<0.05)$ part of the ACL graft.

AMR of the proximal ACL portion rose significantly from 6 weeks to 6 months $(p<0.001)$ after surgery and was by then significantly different to a native ACL $(p<0.001)$. Subsequently, the AMR started to decline to reach a similar AMR of the native proximal ACL at 1 and 2 years.

The mid-substance AMR increased significantly from 6 weeks to 6 months postoperatively $(p<0.001)$ before decreasing at 1 year and 2 years postoperatively $(p<0.01)$. The AMR was significant higher at 3- and 6 months after ACL-R compared with a native ACL $(p<0.001)$ but was not different from a native mid-substance ACL at 1 and 2 years.

The distal AMR rose significantly between 6 weeks and 6 months postoperatively $(p<0.05)$. It then dropped slowly to the lowest AMR at 2 years postoperatively. AMR of the distal intraarticular graft section was significantly lower than a native ACL at 2 years after surgery $(p<0.05$, Fig. 3$)$.

\section{Correlation to clinical outcome, knee stability, and return to sports}

Anterior knee laxity measured by KT-1000 device was $1.9 \pm 1.7 \mathrm{~mm}$ and $2.4 \pm 2.3 \mathrm{~mm}$ higher compared to the healthy contralateral knee at 1 and 2 years postoperatively, respectively. For neither APR nor AMR a significant correlation with KT-1000 measurements at the respective time points could be identified.
IKDC and Lysholm score improved significantly from preoperative values to postoperative values at 6 months, 1-, and 2 years postoperatively (all $p<0.001$, Fig. 4 ). There was also a significant increase in clinical outcome scores between 6 months and 1 year postoperatively but not from 1 to 2 years (IKDC: $p<0.001$, Lysholm score $p<0.05$ ). APR and AMR were not associated with IKDC and Lysholm score at any time point.

\section{Return to preinjury sports levels}

$94 \%(16 / 17)$ of the patients returned to sports within 2 years after ACL-R. One patient suffered a contralateral ACL injury 1.5 years after initial ACL-R and could therefore not RTS at final follow-up. Median TAS was 6 (range 4-9) before injury, 4 (range 3-6) after 6 months, 5 (range 1-7) after 1 year and 6 (range 1-9) at final follow up. At 1 year and 2 years follow-up, 41\% (7/17 patients) and 65\% (11/17) achieved the preinjury TAS level, respectively. TAS was not correlated with APR or AMR at any time point within the small patients' cohort. However, APR and AMR of the ACL mid-substance were significantly lower (hypo-intense) in patients who could return to the preinjury sports level compared to those who did not achieve the same sports level $(p<0.05)$.

\section{Discussion}

The most important findings of our study were that an ACL autograft is undergoing a significant transformation process during the first 2 years postoperatively which is consistent
Fig. 4 Patient reported outcome scores (Subjective IKDC and Lysholm score) improved significantly from preoperative to 6 months, $1-$, and 2 years after ACL reconstruction

\section{Clinical outcome scores}

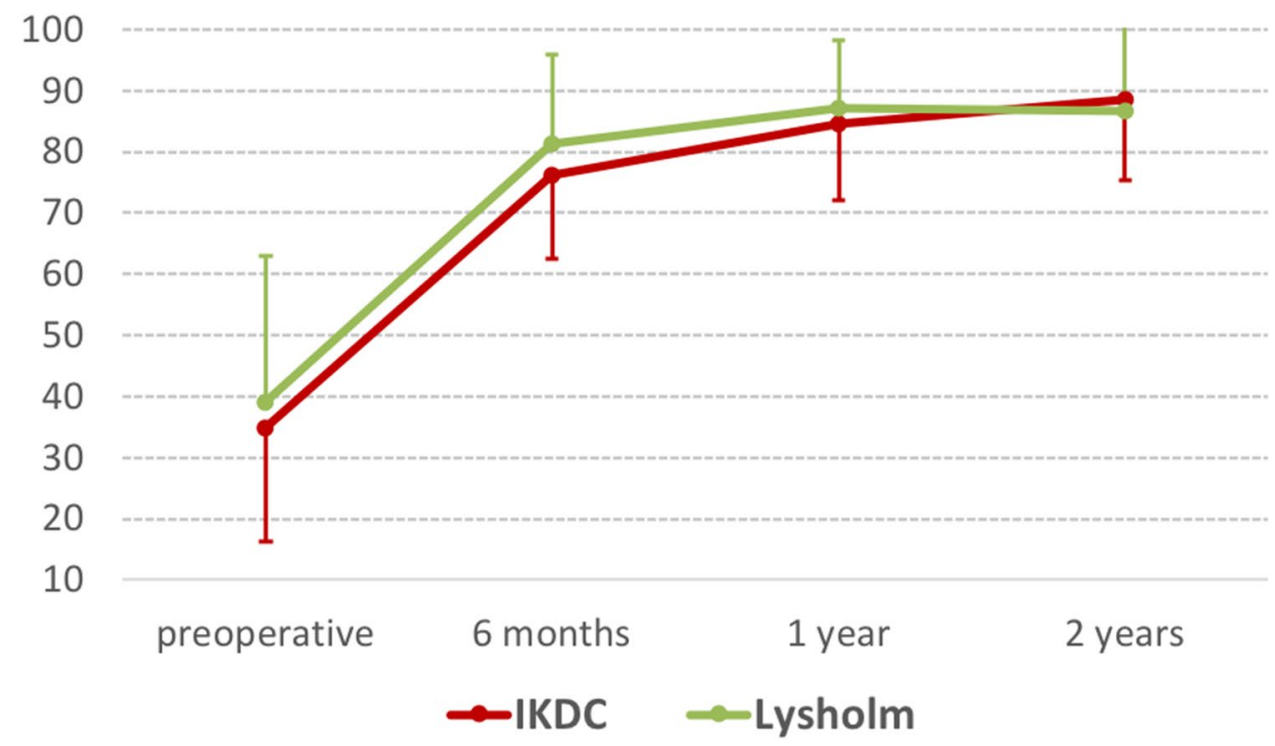


with the previously described ligamentization process [9, $20,45]$. These findings confirmed our first hypothesis that an ACL graft exhibits a high MRI signal intensity 6 months after surgery but approximates the MRI appearance of a native ACL at 1- and 2 years postoperatively. Hypointense MRI signal (lower APR or AMR) did not correlate with higher clinical outcome scores and lower anterior knee laxity what contradicted our second hypothesis. However, patients with lower mid-substance ACL signal intensity were more likely to return to the preinjury sports level.

It is known that $\mathrm{T} 2$ relaxation time is correlated to tissue water content and collagen orientation, with higher values indicating greater disorganization and lower values representing less disorganization [5, 9]. After ACL-R, values are expected to increase due to graft remodelling followed by a subsequent reduction [16, 42, 45]. Accordingly, changes in MRI signal of the ACL graft can be observed due to ultrastructural changes of graft over time. Previously, research on ACL autograft maturation used different MRI measurement methods, such as the signal/noise quotient (SNQ) [6, $8,12,17,21,26,38,48]$, signal intensity ratio (SIR) [11], or T2 mapping [3, 9, 16, 24, 45]. In the present study, the graft signal intensity on MRI was normalized to the subjectspecific signal intensity of both the PCL and the gastrocnemius muscle in order to minimize inter-scan variability. Muscle tissue was chosen as a second internal reference to avoid systematic measurement error caused by variation due to possible degenerative changes of the PCL. It was concluded that not including the measurement of background noise (proton density of extracorporeal hydrogen atoms) the reliability and accuracy was increased. This assumption is based on the experience of the two readers, which showed highly variable background noise between subjects and the location of ROI.

ACL grafts underlie a histological healing process that includes initial avascular necrosis, revascularization, resynovialization, and remodelling [2, 10, 14, 29, 47]. Previous research revealed that the revascularization process influences the ACL graft signal intensity in MRI with signal intensity being significantly associated with time from surgery [18, 25, 27, 38]. In line with previous reports, the graft signal intensity in the present study peaks at 6 months, followed by a constant decrease [9, 20,45]. In agreement with the results of Chu and Williams [9], the present study suggests an approximation of ACL graft signal intensity to the MRI signal of a native ACL 1 - and 2 years postoperatively indicating graft maturity.

More specifically, the native ACL and the ACL autografts demonstrated regional differences in MRI signal intensity. It was observed that distal regions of native ACL exhibited higher APR and AMR (hyper-intense signal) compared to proximal or mid-substance regions (Fig. 3F). In contrast, the distal ACL autograft showed lower APR and AMR (hypo-intense signal) compared to the mid-substance in the early healing phase and this phenomenon was also observed in former studies [20, 21, 38, 45]. Tashiro et al. found higher MRI signal in the proximal and mid-substance part of the ACL graft compared to the distal graft at 6 months postoperatively [38]. The authors assumed that a high graft bending angle at the femoral drill hole contributes to increased graft signals due to elevated tension after footstrike [38]. Ma et al. [21] found also the highest MRI signal in the proximal ACL graft and thought that graft length change patterns during flexion, femoral fixation method, and the angle of the femoral tunnel are contributing factors for a longer maturation process proximally. The proximal and mid-substance section of the ACL autograft seems to exhibit a higher collagen disorganization in the remodelling phase compared to the distal part [20, 21, 38], and is, hence, presumably more vulnerable for re-injury.

To avoid early biological failures, some studies tried to find correlations between MRI findings and functional and clinical outcomes lately. In previous reports, MRI- and KT-1000 measurements after ACL-R were either poorly or not correlated $[11,12]$. In agreement with the results of Hakozaki et al. [11], no significant correlation between APR or AMR and KT-1000 measurements was identified after 12 or 24 months in the present study. These findings emphasize the importance of a clinical laxity assessment to ensure knee stability before allowing the patient return to sports.

Furthermore, no correlation between APR/AMR and clinical scores could be found in the present study. These results are further strengthened by a study of Li et al. [17], who were not able to show a significant association between ACL autograft SNQ and IKDC, Lysholm score, and TAS. Most patients (94\%) of the present study were able to return to sports and $65 \%$ of these recreational athletes were able to achieve the same sports level. Interestingly, lower APR/ AMR of the ACL mid-substance part increased the chance of returning to the same preinjury sports level 2 years after surgery. This finding is supported by the results of $\mathrm{Li}$ et al. [18], who were able to show a significant positive association between TAS and SNQ values in male subjects.

This study has several limitations. Firstly, histologic data are missing. Therefore, the transformation process of a reconstructed ACL in the first 2 years postoperatively was only described on the basis of MRI examinations. Secondly, only males were included in the ACL-R group so the results cannot be transferred for ACL ligamentization in females. Thirdly, even though the number of patients in this study was low, the statistical power of the current study was 0.87 . Considering six MRI scans per patient, the number of subjects was intended to be narrowly restricted to minimize the number of scans and costs. Lastly, the measurement methods (APR, AMR) used in this study have not been used before. Nevertheless, the authors believe that by using two different 
ratios and by not including background noise measurements the drawback was overcome.

The results of the present study provide a template for monitoring the normal ACL maturation process via MRI in case of prolonged clinical symptoms. However, subjective outcome and clinical examination of knee laxity remain important to assess the treatment success and to allow to return to sports.

\section{Conclusion}

ACL grafts undergo a continuous maturation process in the first 2 years after surgery. The ACL graft signals became hyper-intense 6 months postoperatively and approximate the signal of a native intact ACL at 12- and 24 months. ACL autograft signal intensity exhibits significant differences between the mid-substance and distal portion in the early healing phase. Patients with a hypo-intense ACL graft signal at 2 years follow-up were more likely to return to the preoperative sports level.

Author contributions All listed authors have contributed substantially to this work (PML, LW, KW, and AA for the study conception and design; PML, LW, and VS for the data collection; LW and PML for the data analysis; LW, PML, KW, and ABI for the data interpretation; PML, LW, and AA for the drafting of the manuscript, the figures, and the literature research; PML, AA, VS, KW, ABI, and LW for critical revising the manuscript in terms of intellectual and professional input) and have approved the submission to Knee Surgery, Sports Traumatology, Arthroscopy (KSSTA).

Funding Open Access funding enabled and organized by Projekt DEAL. No funding was received.

\section{Declarations}

Conflict of interest Andreas B. Imhoff is a consultant for Arthrosurface and Medi Bayreuth and receives royalties from Arthrex and Arthrosurface. All other authors declare that they have no conflict of interest related to this study.

Ethical approval Ethical approval was obtained from the Ethics Committee of the Technical University Munich (329/19S). All procedures performed were in accordance with the ethical standards of the institutional and/or national research committee and with the 1964 Declaration of Helsinki and its later amendments or comparable ethical standards.

Informed consent All subjects gave their written informed consent to participate in this investigation.

Open Access This article is licensed under a Creative Commons Attribution 4.0 International License, which permits use, sharing, adaptation, distribution and reproduction in any medium or format, as long as you give appropriate credit to the original author(s) and the source, provide a link to the Creative Commons licence, and indicate if changes were made. The images or other third party material in this article are included in the article's Creative Commons licence, unless indicated otherwise in a credit line to the material. If material is not included in the article's Creative Commons licence and your intended use is not permitted by statutory regulation or exceeds the permitted use, you will need to obtain permission directly from the copyright holder. To view a copy of this licence, visit http://creativecommons.org/licenses/by/4.0/.

\section{References}

1. Abe S, Kurosaka M, Iguchi T, Yoshiya S, Hirohata K (1993) Light and electron microscopic study of remodeling and maturation process in autogenous graft for anterior cruciate ligament reconstruction. Arthroscopy 9:394-405

2. Amiel D, Kleiner JB, Roux RD, Harwood FL, Akeson WH (1986) The phenomenon of "ligamentization": anterior cruciate ligament reconstruction with autogenous patellar tendon. J Orthop Res 4:162-172

3. Anz AW, Edison J, Denney TS, Branch EA, Walz CR, Brock KV et al (2020) 3-T MRI mapping is a valid in vivo method of quantitatively evaluating the anterior cruciate ligament: rater reliability and comparison across age. Skeletal Radiol 49:443-452

4. Ardern CL, Taylor NF, Feller JA, Webster KE (2014) Fifty-five per cent return to competitive sport following anterior cruciate ligament reconstruction surgery: an updated systematic review and meta-analysis including aspects of physical functioning and contextual factors. Br J Sports Med 48:1543-1552

5. Biercevicz AM, Miranda DL, Machan JT, Murray MM, Fleming BC (2013) In Situ, noninvasive, T2*-weighted MRI-derived parameters predict ex vivo structural properties of an anterior cruciate ligament reconstruction or bioenhanced primary repair in a porcine model. Am J Sports Med 41:560-566

6. Bouguennec N, Robinson J, Douiri A, Graveleau N, Colombet PD (2021) Two-year postoperative MRI appearances of anterior cruciate ligament hamstrings autografts are not correlated with functional outcomes, anterior laxity, or patient age. Bone Joint Open 2:569-575

7. Çelik D, Çoban Ö, Kılıçoğlu Ö (2019) Minimal clinically important difference of commonly used hip-, knee-, foot-, and anklespecific questionnaires: a systematic review. J Clin Epidemiol 113:44-57

8. Chen L, Wu Y, Lin G, Wei P, Ye Z, Wang Y et al (2018) Graft bending angle affects allograft tendon maturity early after anterior cruciate ligament reconstruction. Knee Surg Sports Traumatol Arthrosc 26:3048-3054

9. Chu CR, Williams AA (2019) Quantitative MRI UTE-T2* and $\mathrm{T} 2 *$ show progressive and continued graft maturation over 2 years in human patients after anterior cruciate ligament reconstruction. Orthop J Sports Med 7:2325967119863056

10. Falconiero RP, DiStefano VJ, Cook TM (1998) Revascularization and ligamentization of autogenous anterior cruciate ligament grafts in humans. Arthroscopy 14:197-205

11. Hakozaki A, Niki Y, Enomoto H, Toyama Y, Suda Y (2015) Clinical significance of $\mathrm{T} 2 *$-weighted gradient-echo MRI to monitor graft maturation over one year after anatomic double-bundle anterior cruciate ligament reconstruction: a comparative study with proton density-weighted MRI. Knee 22:4-10

12. Hofbauer M, Soldati F, Szomolanyi P, Trattnig S, Bartolucci F, Fu F et al (2019) Hamstring tendon autografts do not show complete graft maturity 6 months postoperatively after anterior cruciate ligament reconstruction. Knee Surg Sports Traumatol Arthrosc 27:130-136 
13. IKDC (2000) Formblätter International Knee Documentation Committee. https://www.sportsmed.org/AOSSMIMIS/membe rs/downloads/research/IKDCGerman.pdf

14. Janssen RP, van der Wijk J, Fiedler A, Schmidt T, Sala HA, Scheffler SU (2011) Remodelling of human hamstring autografts after anterior cruciate ligament reconstruction. Knee Surg Sports Traumatol Arthrosc 19:1299-1306

15. Kim SH, Lee JW, Kim SG, Cho HW, Bae JH (2021) Low rate of return to preinjury Tegner activity level among recreational athletes: results at 1 year after primary ACL reconstruction. Orthop J Sports Med 9:2325967120975751

16. Lansdown DA, Xiao W, Zhang AL, Allen CR, Feeley BT, Li X et al (2020) Quantitative imaging of anterior cruciate ligament (ACL) graft demonstrates longitudinal compositional changes and relationships with clinical outcomes at 2 years after ACL reconstruction. J Orthop Res 38:1289-1295

17. Li H, Chen J, Li H, Wu Z, Chen S (2017) MRI-based ACL graft maturity does not predict clinical and functional outcomes during the first year after ACL reconstruction. Knee Surg Sports Traumatol Arthrosc 25:3171-3178

18. Li H, Chen S, Tao H, Li H, Chen S (2014) Correlation analysis of potential factors influencing graft maturity after anterior cruciate ligament reconstruction. Orthop J Sports Med 2:2325967114553552

19. Lindanger L, Strand T, Mølster AO, Solheim E, Inderhaug E (2019) Return to play and long-term Participation in pivoting sports after anterior cruciate ligament reconstruction. Am J Sports Med 47:3339-3346

20. Liu S, Li H, Tao H, Sun Y, Chen S, Chen J (2018) A randomized clinical trial to evaluate attached hamstring anterior cruciate ligament graft maturity with magnetic resonance imaging. Am J Sports Med 46:1143-1149

21. Ma Y, Murawski CD, Rahnemai-Azar AA, Maldjian C, Lynch $\mathrm{AD}, \mathrm{Fu} \mathrm{FH}$ (2015) Graft maturity of the reconstructed anterior cruciate ligament 6 months postoperatively: a magnetic resonance imaging evaluation of quadriceps tendon with bone block and hamstring tendon autografts. Knee Surg Sports Traumatol Arthrose 23:661-668

22. Mardani-Kivi M, Azari Z, Hasannejad F (2020) Return to sport activity after anterior cruciate ligament reconstruction: a 6-10 years follow-up. J Clin Orthop Trauma 11:S319-s325

23. Nagelli CV, Hewett TE (2017) Should return to sport be delayed until 2 years after anterior cruciate ligament reconstruction? Biological and functional considerations. Sports Med 47:221-232

24. Niki Y, Yasuoka T, Kobayashi S, Harato K, Nagura T, Okuda S et al (2019) Feasibility of T1rho and T2 map magnetic resonance imaging for evaluating graft maturation after anatomic doublebundle anterior cruciate ligament reconstruction. J Orthop Surg Res 14:140

25. Ntoulia A, Papadopoulou F, Zampeli F, Ristanis S, Argyropoulou M, Georgoulis A (2013) Evaluation with contrast-enhanced magnetic resonance imaging of the anterior cruciate ligament graft during its healing process: a two-year prospective study. Skeletal Radiol 42:541-552

26. Oshima T, Putnis S, Grasso S, Klasan A, Parker DA (2020) Graft size and orientation within the femoral notch affect graft healing at 1 year after anterior cruciate ligament reconstruction. Am J Sports Med 48:99-108

27. Panos JA, Webster KE, Hewett TE (2020) Anterior cruciate ligament grafts display differential maturation patterns on magnetic resonance imaging following reconstruction: a systematic review. Knee Surg Sports Traumatol Arthrosc 28:2124-2138

28. Pauvert A, Robert H, Gicquel P, Graveleau N, Pujol N, Chotel F et al (2018) MRI study of the ligamentization of ACL grafts in children with open growth plates. Orthop Traumatol Surg Res 104:S161-s167
29. Pauzenberger L, Syré S, Schurz M (2013) "Ligamentization" in hamstring tendon grafts after anterior cruciate ligament reconstruction: a systematic review of the literature and a glimpse into the future. Arthroscopy 29:1712-1721

30. Perrone GS, Webster KE, Imbriaco C, Portilla GM, Vairagade A, Murray MM et al (2019) Risk of secondary ACL injury in adolescents prescribed functional bracing after ACL reconstruction. Orthop J Sports Med 7:2325967119879880

31. Putnis S, Neri T, Grasso S, Linklater J, Fritsch B, Parker D (2020) ACL hamstring grafts fixed using adjustable cortical suspension in both the femur and tibia demonstrate healing and integration on MRI at one year. Knee Surg Sports Traumatol Arthrosc 28:906-914

32. Rahardja R, Zhu M, Love H, Clatworthy MG, Monk AP, Young SW (2020) Rates of revision and surgeon-reported graft rupture following ACL reconstruction: early results from the New Zealand ACL registry. Knee Surg Sports Traumatol Arthrosc 28:2194-2202

33. Rougraff B, Shelbourne KD, Gerth PK, Warner J (1993) Arthroscopic and histologic analysis of human patellar tendon autografts used for anterior cruciate ligament reconstruction. Am J Sports Med 21:277-284

34. Sánchez M, Anitua E, Azofra J, Prado R, Muruzabal F, Andia I (2010) Ligamentization of tendon grafts treated with an endogenous preparation rich in growth factors: gross morphology and histology. Arthroscopy 26:470-480

35. Saupe N, White LM, Chiavaras MM, Essue J, Weller I, Kunz M et al (2008) Anterior cruciate ligament reconstruction grafts: MR imaging features at long-term follow-up-correlation with functional and clinical evaluation. Radiology 249:581-590

36. Scheffler SU, Unterhauser FN, Weiler A (2008) Graft remodeling and ligamentization after cruciate ligament reconstruction. Knee Surg Sports Traumatol Arthrosc 16:834-842

37. Snaebjörnsson T, Svantesson E, Sundemo D, Westin O, Sansone M, Engebretsen L et al (2019) Young age and high BMI are predictors of early revision surgery after primary anterior cruciate ligament reconstruction: a cohort study from the Swedish and Norwegian knee ligament registries based on 30,747 patients. Knee Surg Sports Traumatol Arthrosc 27:3583-3591

38. Tashiro Y, Gale T, Sundaram V, Nagai K, Irrgang JJ, Anderst W et al (2017) The graft bending angle can affect early graft healing after anterior cruciate ligament reconstruction: in vivo analysis with 2 years' follow-up. Am J Sports Med 45:1829-1836

39. Tegner Y, Lysholm J (1985) Rating systems in the evaluation of knee ligament injuries. Clin Orthop Relat Res 198:43-49

40. Titchenal MR, Chu CR, Erhart-Hledik JC, Andriacchi TP (2017) Early changes in knee center of rotation during walking after anterior cruciate ligament reconstruction correlate with later changes in patient-reported outcomes. Am J Sports Med 45:915-921

41. Tohyama H, Yasuda K (2000) Extrinsic cell infiltration and revascularization accelerate mechanical deterioration of the patellar tendon after fibroblast necrosis. J Biomech Eng 122:594-599

42. van der List JP, Mintz DN, DiFelice GS (2019) Postoperative magnetic resonance imaging following arthroscopic primary anterior cruciate ligament repair. Adv Orthop 2019:5940195

43. Van Dyck P, Zazulia K, Smekens C, Heusdens CHW, Janssens T, Sijbers J (2019) Assessment of anterior cruciate ligament graft maturity with conventional magnetic resonance imaging: a systematic literature review. Orthop J Sports Med 7:2325967119849012

44. van Groningen B, van der Steen MC, Janssen DM, van Rhijn LW, van der Linden AN, Janssen RPA (2020) Assessment of graft maturity after anterior cruciate ligament reconstruction using autografts: a systematic review of biopsy and magnetic resonance imaging studies. Arthrosc Sports Med Rehabil 2:e377-e388 
45. Warth RJ, Zandiyeh P, Rao M, Gabr RE, Tashman S, Kumaravel $M$ et al (2020) Quantitative assessment of in vivo human anterior cruciate ligament autograft remodeling: a 3-dimensional UTE-T2* imaging study. Am J Sports Med 48:2939-2947

46. Weiler A, Peters G, Mäurer J, Unterhauser FN, Südkamp NP (2001) Biomechanical properties and vascularity of an anterior cruciate ligament graft can be predicted by contrast-enhanced magnetic resonance imaging. A two-year study in sheep. Am J Sports Med 29:751-761

47. Zaffagnini S, De Pasquale V, Marchesini Reggiani L, Russo A, Agati P, Bacchelli B et al (2010) Electron microscopy of the remodelling process in hamstring tendon used as ACL graft. Knee Surg Sports Traumatol Arthrosc 18:1052-1058. https://www.sport
smed.org/AOSSMIMIS/members/downloads/research/IKDCG erman.pdf

48. Zhang Y, Liu S, Chen Q, Hu Y, Sun Y, Chen J (2020) Maturity progression of the entire anterior cruciate ligament graft of insertion-preserved hamstring tendons by 5 years: a prospective randomized controlled study based on magnetic resonance imaging evaluation. Am J Sports Med 48:2970-2977

Publisher's Note Springer Nature remains neutral with regard to jurisdictional claims in published maps and institutional affiliations.

\section{Authors and Affiliations}

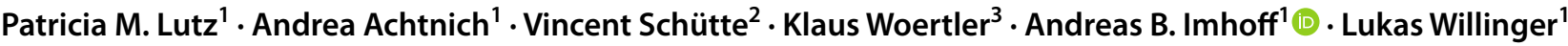

1 Department for Orthopedic Sports Medicine, Technical University Munich, Ismaninger Strasse 22, 81675 Munich, Germany

2 Department for Orthopedic and Trauma Surgery, Martin-Luther-University Halle-Wittenberg, Ernst-Grube-Strasse 40, 06120 Halle (Saale), Germany
3 Department of Diagnostic and Interventional Radiology, Technical University of Munich, Ismaninger Strasse 22, 81675 Munich, Germany 